\title{
A Compact Silicon-on-Insulator Polarization Splitter
}

\author{
Isa Kiyat, Student Member, IEEE, Atilla Aydinli, Member, IEEE, and Nadir Dagli, Member, IEEE
}

\begin{abstract}
A compact directional coupler-based polarization splitter is designed and realized using silicon-on-insulator (SOI) waveguides. Even though silicon does not have any material birefringence, the high index contrast obtained in the SOI platform and reduced waveguide dimensions makes it possible to induce significant birefringence. Polarization splitting is achieved by making use of this geometry-induced birefringence. In this work, we demonstrate polarization splitting in devices as short as $120 \mu \mathrm{m}$. Even smaller devices can be made using submicron-thick Si waveguides.
\end{abstract}

Index Terms-Birefringence, directional couplers, integrated optics, polarization splitters, silicon-on-insulator (SOI) technology.

\section{INTRODUCTION}

$\mathbf{P}$ OLARIZATION splitters find applications in optical systems where polarization states of light are important. Some of these systems are used in communications, sensing, data storage, imaging, and signal processing [1], [2]. For many birefringent optical devices, separation of orthogonal polarization states is a straightforward solution where polarization splitters can be used. So far, directional couplers, asymmetrical Y-junction structures, multimode interference couplers have been adopted as polarization splitting components. These devices have been realized on silica, $\mathrm{LiNbO}_{3}$, GaAs-GaAlAs, InGaAsP-InP, and polymer materials [1]-[4]. Material birefringence, stress-induced birefringence in ion exchanged waveguides, and selective attenuation of orthogonal polarization states using metal over a waveguide have been employed in these polarization splitters. A polarization splitter based on two-dimensional grating coupler etched in a 220-nm-thick SOI waveguide has also been realized [5]. Even microoptoelectromechanical systems have been used for fabrication of polarization separators [4].

In this letter, we report on design and fabrication of an SOI directional coupler utilized as a passive polarization mode splitter. SOI integrated optical directional couplers have already been fabricated [6]. However, they have not been exploited for polarization splitting applications. We make use of birefringence accumulation in SOI rib waveguides as their sizes are reduced. To the best of our knowledge, this device is the first integrated optical polarization splitter based on SOI technology to separate light into transverse-electric (TE) and transverse-magnetic (TM) modes.

Manuscript received August 13, 2004; revised September 4, 2004. This work was supported by a Bilkent University Research Grant (Code: Phys03-02).

I. Kiyat and A. Aydinli are with Bilkent University, Department of Physics, Ankara 06800, Turkey (e-mail: kiyat@fen.bilkent.edu.tr; aydinli@fen.bilkent.edu.tr).

N. Dagli is with Department of Electrical and Computer Engineering, University of California, Santa Barbara, CA 93106 USA (e-mail: dagli@ece.ucsb.edu).

Digital Object Identifier 10.1109/LPT.2004.838133

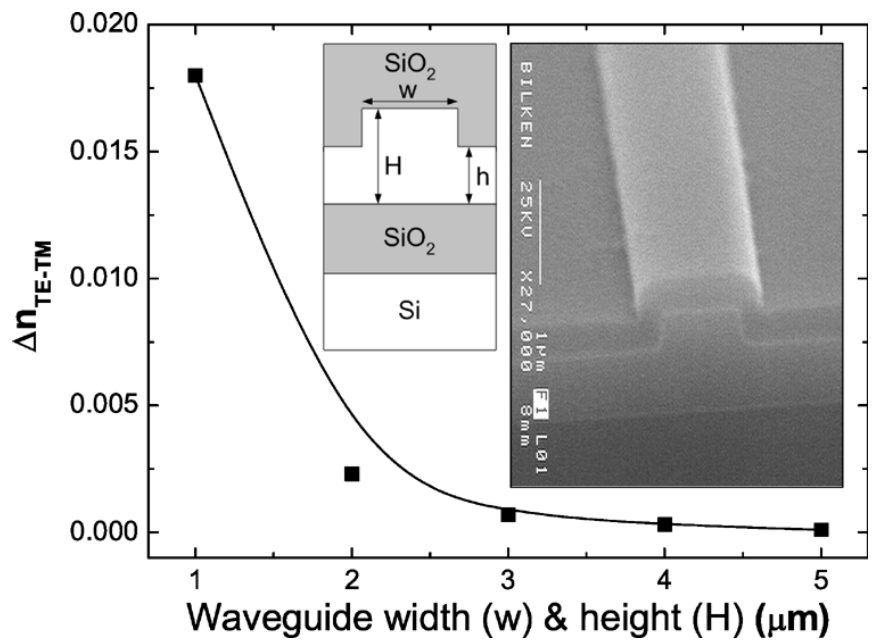

Fig. 1. Effective index difference between TE and TM modes of SM SOI waveguides with $h / H=0.6$. Birefringence increases as waveguide cross-sectional dimensions reduce in size. The insets show critical waveguide dimensions and cross-sectional scanning electron micrograph of a fabricated waveguide.

In Section II, we explain the design and operation principles of the device. Fabrication procedures and measurement techniques follow. Finally, the experimental results and analysis are given.

\section{DESIGN}

The polarization-dependent behavior of integrated optical waveguides has two main sources. One source is the intrinsic material birefringence which can be due to stress in the waveguides. The other source is due to waveguide geometry or cross-sectional profile. SOI single-mode (SM) waveguides with a large cross section are generally known for their polarization insensitivity due to inherent index isotropy of the silicon crystal and negligible stress formed by wafer bonding. The birefringence observed in these waveguides is negligible and has been attributed to cross-sectional geometry of the waveguides [7]. It has been shown by numerical simulation that even the small birefringence in the rib waveguides can be tailored to zero by adjusting the geometrical cross section [8]. However, as cross-sectional dimensions reduce, birefringence can increase and such waveguides can be designed for a specific birefringence value. Analysis of SOI waveguides by three-dimensional (3-D) vector beam propagation (BPM) simulations confirm the evolution of geometrical birefringence in SM SOI waveguides as their size is reduced, as seen in Fig. 1. The inset shows the critical parameters of the waveguide structure. The waveguides with sizes $w, H \leq 1 \mu \mathrm{m}$ display significantly different effective indexes for their TE and TM modes. In our design, width $(w)$ and height $(H)$ of the SM waveguide is chosen to be $1 \mu \mathrm{m}$, 


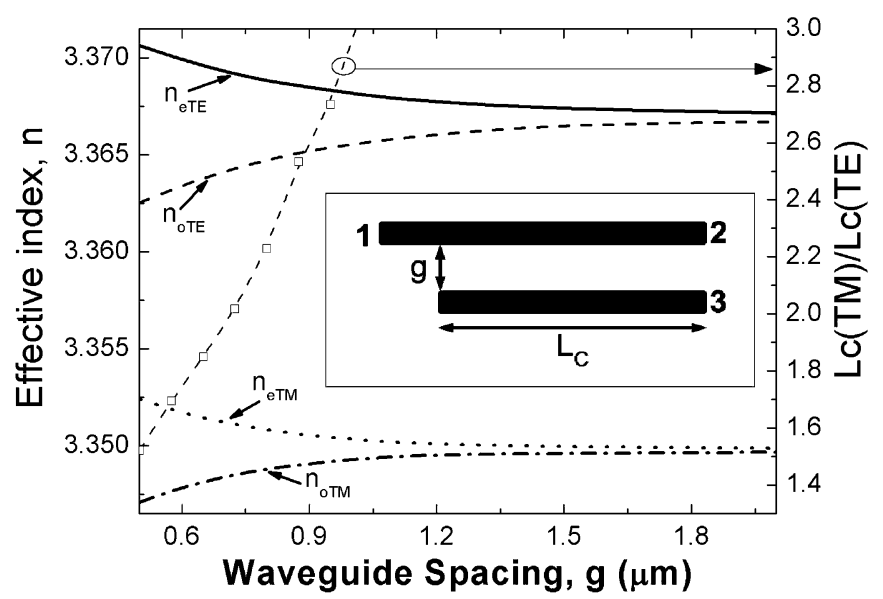

Fig. 2. Effective indexes of even and odd modes of TE and TM modes of a coupler as a function of waveguide spacing $g$. Note that as $g$ increases odd and even propagation constants converge to respective refractive indexes of the isolated waveguides. Inset shows the layout of a directional coupler. The ratio of TM to TE coupling lengths are also shown.

while slab height $(h)$ is $0.6 \mu \mathrm{m}$. These dimensions satisfy the SM condition for an SOI waveguide [9] and can be fabricated with good accuracy.

In the light of this discussion, we design a directional coupler to be used as a polarization splitter using SOI SM waveguides with top silicon layer thickness of $1.0 \mu \mathrm{m}$. For the preliminary design of the polarization splitter, we first consider the device layout shown in the inset of Fig. 2. This is a straight directional coupler which can be analyzed with minimal computational effort. Light is launched into Port 1 and the output power is detected either from Ports 2 or 3 . Optical propagation in a directional coupler can be expressed in terms of even and odd modes of the coupled waveguide system with effective indexes $n_{e}$ and $n_{o}$. The evolution of the effective index of these modes for TE and TM polarizations are shown in Fig. 2 as a function of the waveguide spacing. From the figure, it is clear that for small waveguide spacings, the difference between propagation constants of the odd and even modes is large, while they converge to the TE and TM effective indexes of individual waveguides as the spacing increases. The coupling length $L_{c}$ is the length necessary for complete energy transfer and is different for TE and TM polarizations. The coupling length of either TE or TM polarization is given by

$$
L_{c}=\frac{(\pi)}{\left(n_{e}-n_{o}\right) k_{0}}
$$

in terms of free space wavevector $k_{0}$. We seek a design where

$$
\frac{L_{c}(\mathrm{TM})}{L_{c}(\mathrm{TE})}=2 m
$$

relation is met. Here, $m$ is an integer. The coupling length for TM polarization becomes twice that for TE polarization at a gap value of $0.8 \mu \mathrm{m}$ allowing for TE mode injected at the input port to couple back and forth and detected at the output Port 2, while the TM mode couples only once and is detected at the output Port 3. Using these values as a starting point, we considered the design of the polarization splitter shown in Fig. 3(a). In this case, output waveguides are separated at the output using S-bends.

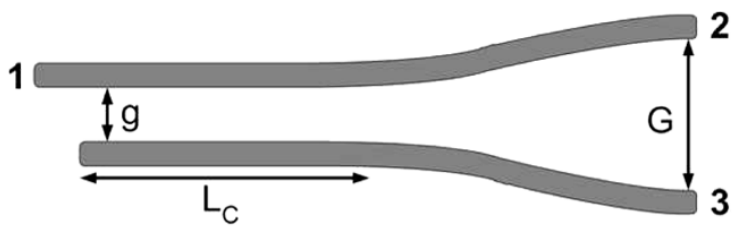

(a)

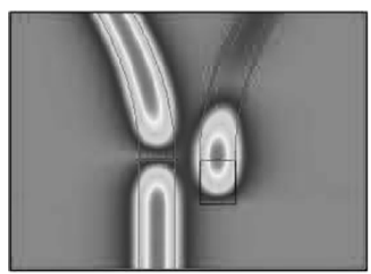

(b)

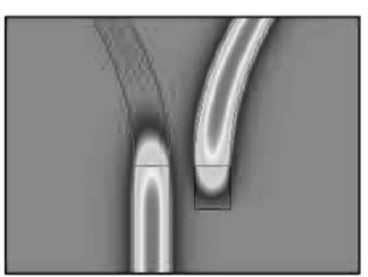

(c)
Fig. 3. Schematic layout of the polarization splitter, (a) with 3-D vector BPM simulation results of a coupler with $g=0.7 \mu \mathrm{m}$ and $L_{c}=110 \mu \mathrm{m}$ for (b) TE and (c) TM modes. The simulations show the excellent polarization splitting. $G$ is $50 \mu \mathrm{m}$ and the length of the S-bend is $3 \mathrm{~mm}$.

In order to take into account the coupling contribution at the S-bend waveguides, full 3-D vector BPM is used to obtain the final design by scanning various waveguide spacings $g$ from 0 to $1.5 \mu \mathrm{m}$. Results of 3-D vector BPM analysis show that gaps of 0.7 and $1.4 \mu \mathrm{m}$ allow polarization splitting with $m$ values of 1 and 2, and $L_{c}(\mathrm{TM})$ lengths of 110 and $2450 \mu \mathrm{m}$, respectively (Fig. 3). The small difference between waveguide spacing obtained from (2) $(0.8 \mu \mathrm{m})$ and that obtained from BPM analysis $(0.7 \mu \mathrm{m})$ is due to the coupling contribution at the $S$-bends. The corresponding devices are the shortest possible polarization splitters with the chosen geometry.

\section{FABRICATION AND MEASUREMENTS}

The SOI polarization splitters are fabricated so that the waveguide widths and heights are $1 \mu \mathrm{m}$. Such small dimensions require very uniform silicon layer thickness. Standard bond and etch back SOI (BESOI) wafers show thickness variations of $500 \mathrm{~nm}$ which is not suitable for our purpose. However, Unibond wafers have thickness uniformities ranging from $\pm 20 \mathrm{~nm}$ down to $\pm 5 \mathrm{~nm} .{ }^{1}$ These wafers are fabricated by $\mathrm{H}^{+}$ion implantation followed by heat treatment, while BESOI wafers are bonded and polished down to the desired thickness.

We start the fabrication with a Unibond wafer with silicon layer thickness of $1000 \pm 20 \mathrm{~nm}$ and cleave a piece that has dimensions of $1.5 \times 1.5 \mathrm{~cm}$. A photoresist layer is spun and the device is optically exposed by lithography. The pattern is transferred to the SOI chip by reactive ion etching (RIE) using $\mathrm{SF}_{6}$ gas. The RIE etch is followed by the deposition of a thin upper cladding layer of $\mathrm{SiO}_{2}$ using plasma-enhanced chemical vapor deposition. In order to obtain optical quality facets for optical measurements, we cleave the chip at the input and output ports. We find that best cleaves are obtained when we thin the $625-\mu \mathrm{m}$-thick substrate down to approximately $100 \mu \mathrm{m}$. This process is performed by wet etching the backside using HNA $\left(\mathrm{HF}, \mathrm{HNO}_{3}\right.$, and $\mathrm{CH}_{3} \mathrm{COOH}$ with ratio $\left.1: 8: 2\right)$ which etches isotropically resulting in smooth surfaces.

\footnotetext{
${ }^{1}$ SOITEC Silicon on Insulator Thechnologies, Bernin, France.
} 


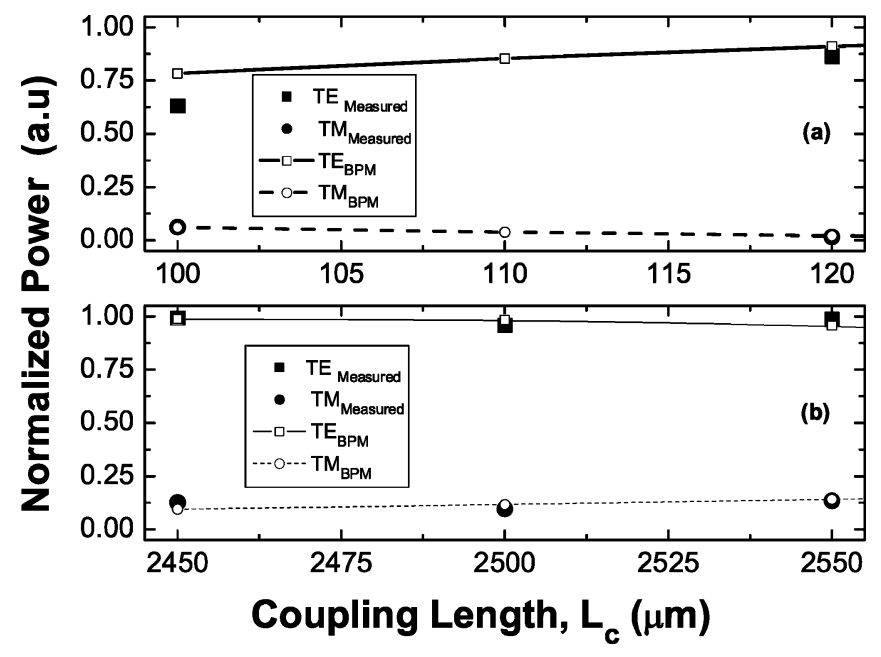

Fig. 4. Normalized polarized optical power measured at the output Port 2 for both TE and TM input signals for (a) gap $=0.7 \mu \mathrm{m}$ and (b) gap $=1.4 \mu \mathrm{m}$. Lines are drawn to guide the eye.

The fabricated and optically cleaved devices are tested on a standard integrated optics setup with a distributed feedback laser operating at $1550 \mathrm{~nm}$. The input light polarization state is controlled by a fiber polarization controller. In order to improve light coupling to the waveguides, a tapered and lensed fiber with beam spot diameter of $2 \mu \mathrm{m}$ was used. TE and TM measurements are made separately. Before each measurement, the laser light optimized in either polarization state is focused on the input facet of the directional coupler. A polarizer with high extinction ratio is used after the device to confirm the polarization state of the measured signal. The input waveguide connected to Port 1 was at least $3.5 \mathrm{~mm}$ from the point where the coupler starts to eliminate the possibility of exciting both waveguides of the coupler while exciting Port 1. Light at the output ports are viewed by an infrared camera and captured by a TV-video card. The results for gaps of 0.7 and $1.4 \mu \mathrm{m}$ are given in Fig. 4. We observe very good polarization splitting at the designed coupling length of $2450 \mu \mathrm{m}$ for $1.4-\mu \mathrm{m}$ gap (extinction ratios of $20.8 \mathrm{~dB}$ for TE and $9.7 \mathrm{~dB}$ for TM polarizations) and at the designed coupling length of $120 \mu \mathrm{m}$ for $0.7-\mu \mathrm{m}$ gap (extinction ratios of $18.1 \mathrm{~dB}$ for TM and $8.0 \mathrm{~dB}$ for TE polarizations). Extinction ratios quoted are defined as $10 \log (P 2 / P 3)$ for TE polarization and $10 \log (P 3 / P 2)$ for TM polarization in terms of $P 2$ and $P 3$, optical powers measured at Ports 2 and 3, respectively. The results obtained from 3-D vector BPM simulations and the results obtained from the experiments are in very good agreement when the coupling contribution from the $\mathrm{S}$-bends is taken into account. The relatively large insertion losses due to small cross-sectional areas of these devices can be alleviated by using mode transformers. Propagation losses of $5.5 \mathrm{~dB} / \mathrm{cm}$ measured on straight waveguides with the same dimensions are in good agreement with the literature [10] and are due to sidewall roughness and can be further reduced by controlled oxidation of the waveguide surfaces [10]. These results prove that geometrical birefringence-based polarization control is possible in the thin core layers of SOI wafers. Even smaller devices can be made using smaller gaps and smaller waveguide dimensions using submicron core layers.

\section{CONCLUSION}

An SOI rib waveguide becomes birefringent as its size is reduced. This idea is used to design and fabricate a directional coupler polarization splitter based on geometrical birefringence. The device uses $1-\mu \mathrm{m}$-sized SOI waveguides. The length of the device is about $2500 \mu \mathrm{m}$ for gap of $1.4 \mu \mathrm{m}$ but is drastically shortened to about $120 \mu \mathrm{m}$ for gap of $0.7 \mu \mathrm{m}$. We, thus, have demonstrated the first polarization splitter that uses geometrical birefringence control of high-index optical waveguides on SOI platform.

\section{REFERENCES}

[1] A. Miliou, R. Srivastava, and R. V. Ramaswamy, "A 1.3- $\mu$ m directional coupler polarization splitter by ion exchange," J. Lightw. Technol., vol. 11, no. 2, pp. 220-225, Feb. 1993.

[2] K. Okamoto, M. Doi, T. Irita, Y. Nakano, and K. Tada, "Fabrication of TE/TM mode splitter using completely buried GaAs/GaAlAs waveguide," Jpn. J. Appl. Phys., vol. 34, no. 1, pp. 114-115, 1990.

[3] P.-K. Wei and W.-S. Wang, "A TE-TM mode splitter on lithium niobate using Ti, $\mathrm{Ni}$, and $\mathrm{MgO}$ diffusions," IEEE Photon. Technol. Lett., vol. 6 , no. 2, pp. 245-248, Feb. 1994.

[4] C. Pu, Z. Zhu, and Y.-H. Lo, "Surface micromachined integrated optic polarization beam splitter," IEEE Photon. Technol. Lett., vol. 10, no. 7, pp. 988-990, Jul. 1998.

[5] D. Taillaert, H. Chong, P. I. Borel, L. H. Frandsen, R. M. De La Rue, and R. Beats, "A compact two-dimensional grating coupler used as a polarization splitter," IEEE Photon. Technol. Lett., vol. 15, no. 9, pp. 1249-1251, Sep. 2003.

[6] P. D. Trinh, S. Yagnanarayanan, and B. Jalali, "Integrated optical directional couplers in silicon-on-insulator," Electron. Lett., vol. 31, no. 24, pp. 2097-2098, 1995.

[7] B. Jalali, S. Yagnanarayanan, T. Yoon, T. Yoshimoto, I. Rendina, and F. Coppinger, "Advances in silicon-on-insulator optoelectronics," IEEE J. Sel. Topics Quantum Electron., vol. 4, no. 6, pp. 938-947, Nov./Dec. 1998.

[8] D. Dai and S. He, "Analysis of the birefringence of a silicon-on-insulator rib waveguide," Appl. Opt., vol. 43, no. 5, pp. 1156-1161, 2004.

[9] R. A. Soref, J. Schmidtchen, and K. Petermann, "Large single-mode rib waveguides in $\mathrm{GeSi}-\mathrm{Si}$ and $\mathrm{Si}-\mathrm{on}-\mathrm{SiO}_{2}$," IEEE J. Quantum Electron., vol. 27, no. 8, pp. 1971-1974, Aug. 1991.

[10] K. K. Lee, D. R. Lim, L. C. Kimerling, J. Shin, and F. Cerrina, "Fabrication of ultralow-loss $\mathrm{Si} / \mathrm{SiO}_{2}$ waveguides by roughness reduction," Opt. Lett., vol. 43, no. 5, pp. 1156-1161, 2004. 